Revue de l'Institut des langues et cultures

d'Europe, Amérique, Afrique, Asie et Australie

$32 \mid 2018$

Didactique des langues et cultures de spécialité : méthodes, corpus et nouvelles technologies

\title{
Création d'activités lexicales basées sur corpus pour la formation des traducteurs
}

Creation of Corpus-Based Lexicon Activities

\section{Marjan Alipour}

\section{OpenEdition}

\section{Journals}

Édition électronique

URL : http://journals.openedition.org/ilcea/4825

DOI : 10.4000/ilcea.4825

ISSN : 2101-0609

Éditeur

UGA Éditions/Université Grenoble Alpes

\section{Édition imprimée}

ISBN : 978-2-37747-059-4

ISSN : 1639-6073

\section{Référence électronique}

Marjan Alipour, «Création d'activités lexicales basées sur corpus pour la formation des traducteurs », ILCEA [En ligne], 32 | 2018, mis en ligne le 01 juillet 2018, consulté le 20 avril 2019. URL : http:// journals.openedition.org/ilcea/4825; DOI : 10.4000/ilcea.4825

Ce document a été généré automatiquement le 20 avril 2019

(C) ILCEA 


\section{Création d'activités lexicales basées sur corpus pour la formation des traducteurs}

Creation of Corpus-Based Lexicon Activities

Marjan Alipour

\section{Introduction}

\subsection{Problématique}

1 Les étudiants en traduction sont amenés à traduire des textes spécialisés liés à des domaines de connaissance différents : juridique, scientifique, par exemple. Chacune de ces disciplines possède son propre lexique, soit le lexique spécialisé (lexS) que les futurs traducteurs, particulièrement ceux désirant se diriger vers la traduction spécialisée, doivent maîtriser pour pouvoir exprimer correctement des connaissances, même s'ils ne sont pas experts du domaine, d'où notre intérêt pour cet élément clé.

2 Certaines études (Kiraly, 2000 ; González Davies, 2004 ; Hurtado Albir, 2008) soutiennent que des approches classiques sont adoptées pour la formation des traducteurs, notamment la pédagogie lisez et traduisez (González Davies, 2004 ; Hurtado Albir, 2008) ${ }^{1}$. Par ailleurs, l'étude d'Echeverri (2008), en plus de notre expérience et nos observations des cours de traduction, révèle que les formules pédagogiques classiques sont appliquées dans les universités canadiennes ${ }^{2}$. Ces pratiques mettent l'enseignant au cœur de toutes les activités en classe : celui-ci donne les solutions aux problèmes; ses commentaires et corrections forment l'ensemble des connaissances de l'apprenant qui joue un rôle passif dans son apprentissage.

3 Par ailleurs, les manuels de traduction spécialisée (Alcaraz \& Hughes, 2014 ; Bédard, 1986 ; Maillot, 1981; Rouleau, 2011) soulignent l'importance de la connaissance lexicale et présentent les difficultés lexicales rencontrées lors d'une traduction, entre autres la polysémie, la synonymie, les faux amis. Par ailleurs, on peut noter que de plus en plus de 
travaux sont effectués sur la traduction spécialisée et la langue de spécialité (Guével, 2007; Maniez \& Thoiron, 2007; Scarpa, 2011). Ces études mettent en avant les particularités des textes spécialisés, notamment la terminologie qu'on y rencontre, et les difficultés rencontrées par les traducteurs (le lexique, la forme, le style, entre autres). Toutefois, ils ne proposent pas de méthodes d'enseignement permettant à l'apprenanttraducteur de résoudre les problèmes lexicaux de façon autonome.

Pour ce qui est du lexique, des publications récentes sur l'enseignement des langues signalent le peu de place qu'on y accorde (Garcia-Debanc, Masseron \& Ronveaux, 2013 ; Grossmann, 2011; Nonnon, 2012). Binon et Verlinde (2004) et Mangiante (2002) mettent l'accent sur l'importance du lexS dans les discours spécialisés, or nos observations des cours de langue de spécialité et nos entretiens avec leurs enseignants révèlent que cet élément clé n'est pas enseigné de façon systématique ${ }^{3}$. En effet, dans ces classes, on se concentre surtout sur le style d'écriture dans les textes spécialisés, ou bien le lexS y est abordé de manière implicite lors de la correction des travaux de traduction. L'accent est alors mis particulièrement sur les erreurs fréquentes (par exemple, tel terme ne se traduit pas par tel terme).

\subsection{Objectifs}

Cet article s'inscrit dans le cadre d'une thèse de doctorat en traduction visant à élaborer des méthodes d'enseignement-apprentissage du lexS pour les étudiants en traduction. Nous nous intéressons particulièrement aux termes liés au domaine de la pollution de l'eau.

6 Nous comptons, d'une part, accompagner les apprenants-traducteurs dans leur apprentissage, et d'autre part, assister les enseignants dans la formation des apprenantstraducteurs en leur proposant des outils et des moyens d'accompagnement de leurs étudiants. Notons que nous nous intéressons à l'enseignement-apprentissage du lexS dans le cadre des cours de langue de spécialité (français) ou de terminologie.

7 Dans la présente étude, nous présentons des activités lexicales que nous avons créées, à partir d'un corpus français, en nous appuyant sur l'approche par tâches (APT). Ces exercices ont pour objectif d'amener l'apprenant à appréhender/distinguer les sens des termes en contexte et à les utiliser correctement dans des situations de communication spécifiques. Nous montrerons comment un apprenant peut parvenir à résoudre les ambiguïtés sémantiques à l'aide de ces tâches par la réflexion de façon autonome. Dans cet article nous nous concentrons sur les problèmes lexicaux, spécifiquement la synonymie et quasi-synonymie.

8 Cet article se divise en cinq sections. Outre l'introduction et la conclusion, la section 2 montre comment un corpus peut être utilisé en enseignement-apprentissage du lexS ; dans la section 3, nous présentons les avantages et les limites de l'APT dans l'enseignement-apprentissage du lexS ; enfin, dans la section 4, nous proposons quelques modèles d'activités lexicales.

\subsection{Conception de la notion de terme}

Comme nous l'avons mentionné, nous nous intéressons au lexS, mais nous nous concentrons spécifiquement sur les termes liés à la pollution de l'eau ${ }^{4}$. Nous nous 
appuyons sur l'optique lexico-sémantique, définis par L'Homme (2004), pour définir un terme: unité lexicale (UL) dotée d'un sens et d'une forme et qui, par ailleurs, peut appartenir aux parties du discours du nom, du verbe, de l'adjectif et de l'adverbe.

L'Homme (2004) propose les critères lexico-sémantiques suivants pour identifier les termes :

1. Lien avec le domaine d'étude. Le sens d'une UL doit être lié à un domaine spécialisé, par exemple, la microbiologie ou les finances.

2. La nature des actants sémantiques. Si les actants sémantiques, soit les participants auxquels fait appel une UL de sens prédicatif, sont eux-mêmes retenus en tant que termes, il est fort probable que l'UL en question soit un terme également. Par exemple dans la phrase, le chlore élimine les bactéries, chlore et bactérie étant des termes liés à la pollution de l'eau, éliminer est un terme également.

3. La parenté morphologique. Les dérivés des UL définies comme termes suivant les critères (1) et (2) sont considérées comme des termes également. Par exemple, si impureté est un terme lié au domaine de la pollution de l'eau, alors impur, pur, purifier et purifiant (qui purifie) sont admis comme des termes aussi.

4. Les relations paradigmatiques. Les UL avec lesquelles un terme retenu selon les critères (1), (2) et (3) partage des liens autres que morphologiques - lien de synonymie, d'antonymie, par exemple - comptent parmi les termes. Par exemple, si cyanobactérie est un terme, on devra admettre également son synonyme cyanophycée.

\section{Le corpus : outil d'apprentissage lexical}

Des travaux sur l'exploitation du corpus en traduction sont menés depuis les années 1990 (Baker, 1999; Bowker \& Pearson, 2002; Kübler, 2003; Zanettin, 2002). L'intérêt de certains auteurs (Boulton \& Tyne, 2014; Cavalla \& Loiseau, 2013; Landure \& Boulton, 2010; Kübler, 2014) pour l'utilisation du corpus dans l'enseignement des langues, notamment du lexique (langue de spécialité et langue générale) est notable également. Pourtant, «malgré toutes les avancées prometteuses et enthousiastes que l'on rencontre dans la littérature [...] malgré le développement de ressources et de corpus » (Kübler, 2014 : 1), l'utilisation du corpus n'est pas une pratique courante. Du moins, dans les universités canadiennes, l'enseignement par le corpus n'est pas une méthode répandue aussi bien dans les cours de traduction que de terminologie ou de langues de spécialité. Il est vrai qu'on aborde la compilation du corpus et l'utilisation des outils, notamment les extracteurs de termes et les concordanciers, mais cette ressource n'est pas exploitée comme outil d'enseignement aux fins de traduction ou de tâches terminologiques. Comment expliquer cette réalité ? Selon Zanettin (2002) et Bowker (2002), l'exploitation du corpus est une tâche qui demande beaucoup de temps de classe. Tyne $(2012: 114)$ souligne le manque de convivialité des logiciels de traitement du corpus. Pour Boulton: «L'un des facteurs-clés semble être le manque d'études évaluatives de l'apport des corpus dans les processus d'apprentissage » (2008: 43). Toutefois des auteurs (Beeby, Rodríguez Inés \& Sánchez-Gijón, 2009; Kübler, 2014; Zanettin, Bernardini \& Stewart, 2014) poursuivent leurs recherches et proposent des méthodes d'exploitation plus efficace pour la traduction. Sans aucun doute, l'utilisation du corpus en enseignement passe par une formation approfondie et spécifique des enseignants et des étudiants comme le propose Kübler (2014). 


\subsection{Utilités du corpus dans l'enseignement-apprentissage lexical}

12 Dans certaines situations, les dictionnaires et les bases de données terminologiques Dictionnaire de l'eau, Dictionnaire Environnement, Grand dictionnaire terminologique (GDT), Termium - ne donnent pas suffisamment d'informations sur les termes, notamment sur la différenciation sémantique ou l'usage des termes dans des contextes spécifiques. Par exemple, on n'obtient pas suffisamment ces genres de renseignements sur les termes épuration et purification liés au domaine de l'eau dans GDT ou Termium ${ }^{5}$. De ce fait, l'apprenant est contraint à consulter plusieurs documents ou textes pour trouver des informations sur les termes. Dans ces cas, le corpus peut devenir une bonne ressource en raison des contextes riches en connaissance qu'il contient, à savoir des contextes qui fournissent d'importantes informations, notamment sur le sens et l'usage des termes (Bowker \& Pearson, 2002; Meyer, 2001). Effectivement, comme mentionné par Grossmann, le corpus « fournit [...] une base empirique à l'observation et à l'étude des phénomènes lexicaux, ce qui explique l'intérêt didactique qu'elle peut présenter » (2011: 176).

13 Le corpus peut être bénéfique aussi bien pour l'apprenant-traducteur que pour l'enseignant. Les apprenants peuvent se servir du corpus comme support pour y rechercher des informations précises notamment sur le sens et l'usage des termes. Ils peuvent procéder à la consultation directe ou indirecte de ce type de ressources (Chambers, 2010; Kübler, 2014). La première permet à l'apprenant de chercher des informations directement dans le corpus et de créer des concordances, dans lesquelles il pourra recueillir des renseignements importants. Pour ce faire, la méthode de l' apprentissage orienté par les données (data-driven learning) s'avère efficace, puisque l'apprenant devient un chercheur actif plutôt qu'un apprenant passif (Johns, 1986, 1991). Dans cette approche, l'apprenant est amené à découvrir les usages réels des termes à travers le contact avec les données du corpus, ce qui favorise son autonomisation (Boulton \& Tyne, 2014). En revanche, l'apprenant n'a pas un accès direct au corpus par la consultation indirecte : l'enseignant lui fournit des contextes (des concordances) et lui montre des pistes pour rechercher des données sur le terme à l'étude. Ainsi, le corpus et l'analyse des contextes aide l'apprenant dans sa démarche d'apprentissage puisque cela contribue à : i) observer et analyser le comportement et l'usage des termes à l'étude ; ii) appréhender/distinguer les sens des termes en contexte ; iii) utiliser le terme adéquat véhiculant un sens précis dans un contexte spécifique.

Pour ce qui est des enseignants, le corpus peut servir de ressource pour construire des exemples basés sur des concordances et créer des activités d'apprentissage. Il est clair que l'instructeur privilégie la consultation directe ou indirecte suivant ses objectifs pédagogiques. Cependant, nous pensons comme Chambers (2010), qu'il est préférable d'utiliser la consultation indirecte au départ. Premièrement, le mode direct nécessite des outils, dont des ordinateurs et des concordanciers pour chaque apprenant-traducteur qui peuvent être difficiles à utiliser ou non conviviaux (Tyne, 2012). En outre, cela demande beaucoup de temps et de moyens financiers. Ensuite, le corpus contient une multitude d'informations dont certaines sont utiles, mais d'autres insignifiantes par rapport aux objectifs d'apprentissage fixés, ce dont un initié n'est pas informé. Avec la consultation indirecte, l'enseignant peut suggérer des pistes d'observation des contextes afin d'y repérer les renseignements pertinents. Une fois que l'apprenant est familiarisé avec les 
méthodes d'analyse des contextes, on applique la méthode directe en le plaçant devant le corpus pour qu'il y fasse ses propres découvertes et acquière des connaissances de façon autonome.

\subsection{Appréhension et distinction des sens des termes}

15 Les contextes riches en connaissance, contenus dans le corpus, contiennent d'importantes données qui, obtenues de façon directe ou indirecte, servent d'indices aidant notamment à saisir le sens des termes et à en connaître l'usage. Pour Tréville (2000), ces indices peuvent être de bons indicateurs pour appréhender/distinguer les sens des termes, à condition qu'ils soient identifiés méthodiquement. Ainsi, nous avons développé des méthodes au moyen desquelles l'apprenant est amené à analyser les contextes afin d'y relever des éléments clés qui peuvent servir d'indices contextuels, aussi connus sous le nom de marqueurs linguistiques (L'Homme, 2004) ou de marqueurs définitoires (Condamines \& Dehaut, 2011) pour appréhender/distinguer les sens des termes. Nous emploierons le terme indice contextuel (IC) à l'instar de Tréville (2000) et de Binon et Verlinde (2004).

Les IC peuvent se présenter sous plusieurs formes (Condamines \& Dehaut, 2011 ; Meyer, 2001 ; Pearson, 1998). La présence d'expressions comme également appelé, aussi connu peuvent être indicateurs des synonymes, de même que la conjonction de coordination ou. Par exemple :

Les cyanobactéries sont des procaryotes photosynthétiques, aussi connue sous le nom

d'algues bleu-vert.

On peut aussi utiliser l'alcool éthylique (ou éthanol) comme carburant.

La combinatoire des termes peut également constituer un IC comme le soulignent Binon et Verlinde (2004). En effet, les collocatifs peuvent être un indicateur de similitudes ou différences sémantiques entre des paires de termes. Pour illustrer, observons les contextes ci-dessous pour distinguer les sens de se reproduire et se multiplier à l'aide des IC ${ }^{6}$

Figure 1. - Indices contextuels de se reproduire et se multiplier.

\begin{tabular}{l|l}
\multicolumn{1}{c|}{ Contextes } & \multicolumn{1}{c}{ IC } \\
\hline (1) Les poissons trop petits doivent pouvoir s'échapper, et avoir le temps & poisson \\
de $\{$ se reproduire\}. & \\
(2) Les cyanobactéries $\{$ se multiplient $\}$ et forment des colonies facilement & cyanobactérie \\
jusqu'à être visibles à l'œeil nu. & \\
(3) Lorsqu'ils se trouvent dans des conditions idéales, les micro- & $\begin{array}{l}\text { micro- } \\
\text { organisme }\end{array}$ \\
organismes peuvent $\{$ se reproduire $\}$ par division cellulaire. & $\begin{array}{l}\text { micro- } \\
\text { organisme } \\
\text { (4) Enfin, les micro-organismes peuvent pénétrer et }\{\text { se multiplier }\} \text { dans le } \\
\text { corps humain. }\end{array}$ \\
(5) Les oiseaux aquatiques $\{$ se reproduisent $\}$ sur les îles côtières. &
\end{tabular}

Si l'apprenant identifie les IC correctement, l'appréhension / la distinction des sens des termes devient plus simple, d'où l'importance de montrer à l'apprenant des pistes d'analyse des contextes, ce qui, comme mentionné ci-dessus, avantage la consultation indirecte à ce stade. Une fois les IC repérés, leur organisation (sous forme de grilles par exemple) aide les apprenants à saisir leurs similitudes/différenciations sémantiques 
(Binon, Dancette \& Verlinde, 1998). Par exemple, pour les contextes (1) à (5) ci-dessus, l'organisation des termes se présente comme suit :

Figure 2. - Grille comparative de se reproduire et se multiplier.

\begin{tabular}{l|l} 
se reproduire & se multiplier \\
\hline $\begin{array}{l}\text { poisson } \\
\text { micro-organisme } \\
\text { oiseau }\end{array}$ & $\begin{array}{l}\text { cyanobactérie } \\
\text { micro-organisme }\end{array}$
\end{tabular}

19 On constate, par ces contextes, que se reproduire s'emploie avec des termes désignant aussi bien des organismes pluricellulaires (poisson, mammifere marin) que des organismes unicellulaires (cyanobactérie, micro-organisme); alors que se multiplier s'utilise avec des termes qui se rapportent uniquement à des organismes unicellulaires (bactérie, cyanobactérie).

\section{Adoption de l'approche par tâches dans la conception des activités lexicales}

20 Comme nous l'avons annoncé ci-dessus, pour concevoir nos activités lexicales, nous nous sommes basés sur l'APT, mais avant de présenter les exercices, nous passons en revue les raisons du choix de cette approche.

21 Considérant les formules pédagogiques classiques pratiquées dans les cours de traduction, principalement en raison du rôle passif que joue l'apprenant dans son apprentissage, des auteurs (González-Davies, 2004 ; Hurtado Albir, 2008) ont mis en avant l'approche par tâches. Ces auteurs se sont inspirés de la pratique de cette approche dans l'enseignement des langues (Nunan, 2004 ; Skehan, 2011 ; Willis, 1996) et l'ont adaptée à la formation des traducteurs. Par ailleurs, Li (2013) a appliqué cette approche dans l'enseignement de la traduction de la langue des affaires.

22 Dans l'APT, l'apprenant accomplit plusieurs tâches préparatoires, c'est-à-dire des tâches d'apprentissage qui aident à saisir des principes ou des stratégies et qui le préparent à la réalisation d'une tâche globale (Hurtado Albir, 2008), par exemple, la création d'un minidictionnaire de l'eau. Chacune des tâches préparatoires est conçue avec un objectif d'apprentissage précis et favorise le développement de compétences spécifiques. Les tâches sont créées de manière à ce qu'elles correspondent à celles effectuées dans le monde réel du traducteur professionnel. L'enseignant, quant à lui, joue le rôle d'accompagnateur qui fournit des ressources facilitant l'apprentissage et propose des pistes de recherche de solutions aux problèmes.

Pour González-Davies une tâche se réfère à :

[...] a chain of activities with the same global aim and a final product. The full completion of a task usually takes up several sessions. In each of these, the activities lead along the same 
path towards the same end. On the way, both procedural (know how) and declarative (know what) knowledge are practised and explored. (2004:23) Skehan, 2011) :

1. Pré-tâche : l'enseignant présente le sujet et la tâche à exécuter et fournit les instructions nécessaires; stimule la réflexion sur le sujet à l'étude; montre des images, des affiches ou des exercices semblables ou encore l'application d'un outil (p. ex. un concordancier); propose quelques pistes de recherche. Quant à l'apprenant, il est amené à effectuer des tâches préalables. Pour donner quelques exemples, l'étudiant peut faire des recherches dans des bases de données terminologiques (GDT, Termium) ; lire de courts textes sur des concepts liés au domaine étudié, puis participer à des échanges sur des concepts avec l'enseignant et ses pairs.

2. Tâche : les apprenants réalisent l'activité individuellement ou en équipe. L'enseignant joue le rôle d'accompagnateur et donne quelques pistes au cas où les apprenants s'éloignent des objectifs de la tâche.

3. Post-tâche: les apprenants partagent leurs solutions aux problèmes avec leurs pairs et l'enseignant qui donne une rétroaction, l'idée étant de les inciter à interagir et à participer à la découverte des solutions. Ensuite, on peut leur demander de faire une présentation PowerPoint, ou rédiger un rapport.

Cette approche se caractérise par le rôle central qu'elle confère à l'apprenant qui est amené à résoudre les problèmes par la réflexion, la recherche et l'interaction avec son enseignant et ses pairs (Li, 2012). Ainsi, l'étudiant participe activement à son apprentissage et au développement de ses connaissances; l'enseignant n'est pas la seule source dont les commentaires et les corrections déterminent les solutions aux différents problèmes (Echeverri, 2008) puisqu'il peut découvrir les solutions à l'aide d'autres sources comme les ouvrages, les ressources terminologiques, Internet et le corpus. En outre, l'APT augmente les aptitudes des apprenants à plusieurs niveaux (Hurtado Albir, 2008) :

- leur capacité d'analyse, de déduction et de génération de nouvelles idées ;

- leurs habiletés de recherche ;

- leur faculté d'adaptation aux nouvelles situations ;

- leur capacité d'appliquer les connaissances dans la pratique.

Cependant, dans la formation des traducteurs, les enseignants doivent structurer leur plan de cours en fonction de la structure du programme offerte par le département et sont tenus d'atteindre les objectifs du cours durant un trimestre de 3 mois. Or, cette approche demande beaucoup d'organisation et de créativité de la part de l'enseignant qui doit constamment générer de nouvelles idées et renouveler ses pratiques, ce qui peut s'avérer difficile à mettre en place dans les classes avec un grand nombre d'étudiants.

Toutefois, nous estimons que les activités que nous avons conçues basées sur corpus peuvent s'intégrer dans le plan de cours des enseignants sans en changer grandement la structure générale. Par ailleurs, elles ne sont pas dévoreuses de temps et peuvent être effectuées au moyen de logiciels simples.

\section{Création d'activités lexicales sur la synonymie}

Comme nous l'avons mentionné ci-dessus, dans cette étude, nous nous intéressons à la synonymie. Afin d'aider les apprenants-traducteurs à appréhender/distinguer les sens des termes en contexte, nous proposons des activités lexicales portant sur ces types de 
relations lexicales. Il convient donc de présenter, auparavant, notre conception de la synonymie, laquelle sera abordée comme une relation entre des unités lexicales.

\subsection{La synonymie : conception et typologie}

Nous rejoignons certains auteurs quant à la division des synonymes (Dubuc, 1985 ; Polguère, 2003; Ploux \& Victorri, 1998) : les synonymes parfaits (exacts) et les synonymes partiels (approximatifs ou quasi-synonymes).

\section{$>$ Les synonymes parfaits}

Paire de termes, T1 et T2, qui : i) appartiennent à la même partie du discours; ii) renvoient au même sens; iii) ont des combinaisons de mots identiques; iv) sont interchangeables dans tous les contextes: en remplaçant $\mathrm{T} 1$ par $\mathrm{T} 2$ et vice versa, on obtient des énoncés sémantiquement équivalents dans tous les contextes. Par exemple, nous considérons éthanol (T1) et alcool éthylique (T2) comme des synonymes appartenant à ce groupe. Pour illustrer :

1) Le bioéthanol E85 est composé à $85 \%$ en volume d'un alcool, l'éthanol < appelé aussi $>$ alcool éthylique.

2) L'éthanol $<$ ou $>$ alcool éthylique de formule chimique $<\mathrm{C} 2 \mathrm{H} 5 \mathrm{OH}>$ est de loin le premier biocarburant au monde.

3) Les vitesses de dénitrification obtenues avec l'éthanol ( $\mathrm{C} 2 \mathrm{H} 5 \mathrm{OH})$ sont légèrement supérieures à celle obtenue avec le méthanol ( $\mathrm{CH} 3 \mathrm{OH})$.

$\Rightarrow$ Les vitesses de dénitrification obtenues avec l'alcool éthylique ( $\mathrm{C} 2 \mathrm{H} 5 \mathrm{OH})$ sont légèrement supérieures à celle obtenue avec le méthanol ( $\mathrm{CH} 3 \mathrm{OH})$.

Dans le contexte (1), l'expression appelé aussi indique que T1 et T2 renvoient au même sens. Le contexte (2) montre que les symboles par lesquels éthanol et alcool éthylique sont représentés sont identiques: $\mathrm{C} 2 \mathrm{H} 5 \mathrm{OH}$. On peut en déduire donc qu'en remplaçant T1 par T2 et vice versa on obtient des énoncés identiques dans tous les contextes. Le contexte (3) est un exemple parmi beaucoup d'autres montrant cette interchangeabilité. De ce fait, ces deux termes sont des synonymes parfaits.

\section{> Les synonymes partiels}

Paire de termes qui : i) appartiennent à la même partie du discours; ii) renvoient approximativement au même sens ; iii) ont plusieurs combinaisons de mots en commun ; iv) ne sont pas interchangeables dans certains contextes: en remplaçant T1 par T2 ou vice versa, on obtient des énoncés sémantiquement différents dans certains contextes. Pour illustrer ces critères, considérons les termes se reproduire (T1) et se multiplier (T2) dans le sens de " produire une descendance » dans les contextes ci-dessous. 
1) Les cyanobactéries \{se multiplient et forment des colonies jusqu'à être visibles facilement à l'ail nu...

2) [...] les poissons trop petits doivent pouvoir s'échapper, et avoir le temps de \{se reproduire\}.

3) Les bactéries ne \{se multiplient\} pas dans l'eau

4) Les oiseaux aquatiques \{se reproduisent\} généralement sur les îles côtières.
$\Rightarrow$ Les cyanobactéries \{se reproduisent\} et forment des colonies jusqu'à être visibles facilement à l'oil nu.

$\Rightarrow *[. .$.$] les poissons trop petits doivent$ pouvoir s'échapper, et avoir le temps de $\{$ se multiplier $\}$.

$\Rightarrow$ Les bactéries ne $\{$ se reproduisent $\}$ pas dans l'eau

*Les oiseaux aquatiques \{se multiplient\} généralement sur les îles côtières. suivant l'APT. Les tâches et activités conçues visent un objectif pédagogique précis ; et chaque tâche est composée de trois étapes : 1 . la pré-tâche ; 2 . la tâche ; 3 . la post-tâche.

\section{Pré-tâche}

L'enseignant présente l'objet d'étude et les objectifs de l'exercice et donne des précisions nécessaires.

- Objet d'étude : la (quasi-)synonymie.

- Objectifs pédagogiques : améliorer la capacité des apprenants à distinguer le sens de termes sémantiquement proches.

- Précision : à effectuer en équipe dans la salle de classe. Matériels disponibles : dictionnaires, Internet, contextes extraits d'un corpus.

- Problème à résoudre : distinguer les sens des termes décontamination (T1) / désinfection (T2) dans les contextes ci-dessous à l'aide des indices contextuels.

À l'étape de la pré-tâche, l'enseignant peut stimuler la réflexion sur le sujet de diverses manières. Il peut, par exemple, proposer un exercice et inviter les étudiants à identifier les IC de nature différente (comme les collocations, les conjonctions de coordination) dans plusieurs contextes. Alors, les étudiants partagent leurs réflexions sur les différences/similitudes sémantiques entre ces termes avec leurs pairs et l'enseignant oralement, ce qui leur donne des pistes pour la résolution de problèmes. 


\section{Exécution de la tâche}

Les étudiants se mettent en équipe et s'attribuent chacun un rôle pour effectuer la tâche. La première démarche est d'analyser les contextes et d'y relever les indices contextuels.

\section{a) Identification des indices contextuels}

Pour trouver les IC, deux questions se posent :

1. Quel est le sens de T1 et T2 ? Élimination de substances nocives d'un milieu.

2. Qu'est-ce qui doit être éliminé ? Les substances nocives qui contaminent l'eau. Les types de contaminants constituent les indices contextuels.

Figure 3. - : Indices contextuels de décontamination et désinfection.

\begin{tabular}{|c|c|}
\hline Contextes & IC \\
\hline $\begin{array}{l}\text { (1) S'il n'est pas possible d'éliminer toute source d'ignition, } \\
\text { ne pas procéder au nettoyage et à la \{décontamination\} } \\
\text { de la matière chimique déversée. }\end{array}$ & matière chimique \\
\hline $\begin{array}{l}\text { (2) De nouvelles analyses le prouvent: les unités de } \\
\text { \{décontamination\} des puits installées depuis quelques } \\
\text { années en Inde ou au Bangladesh pour se débarrasser de } \\
\text { l'arsenic ne fonctionnent pas. }\end{array}$ & arsenic \\
\hline $\begin{array}{l}\text { (3) En filtrant d'importants volumes d'eau de mer, les } \\
\text { coquillages ont la faculté de concentrer les impuretés de } \\
\text { toutes sortes présentes dans l'eau de mer: bactéries ou } \\
\text { parasites divers, mais aussi composés chimiques } \\
\text { polluants. Leur \{décontamination\} est possible par un } \\
\text { séjour en bassins d'épuration. }\end{array}$ & $\begin{array}{l}\text { bactérie } \\
\text { parasite } \\
\text { composé } \\
\text { chimique } \\
\text { polluant }\end{array}$ \\
\hline $\begin{array}{l}\text { (4) La \{désinfection }\} \text { de l'eau est un moyen de traitement } \\
\text { permettant de détruire les germes pathogènes présents } \\
\text { dans l'eau... }\end{array}$ & germe \\
\hline $\begin{array}{l}\text { (5) Suit l'étape de }\{\text { désinfection\} pour éliminer toutes les } \\
\text { bactéries résiduelles et les éventuels virus. } \\
\text { (6) On remarque, de façon générale, que les bactéries sont } \\
\text { les plus sensibles à la \{désinfection\}, alors que les virus } \\
\text { et les parasites sont les plus résistants. }\end{array}$ & $\begin{array}{l}\text { bactérie } \\
\text { bactérie, virus, } \\
\text { parasite }\end{array}$ \\
\hline
\end{tabular}

\section{b) Solution}

À l'aide des IC, les étudiants distinguent les similitudes et les différences sémantiques entre $\mathrm{T} 1$ et $\mathrm{T} 2$ et peuvent alors proposer une solution :

- Le terme décontamination s'emploie pour exprimer l'idée d'éliminer toutes sortes de contaminants (les matières chimiques) et les différentes sortes de micro-organismes (bactérie, parasite) dans ces contextes; alors que désinfection, s'utilise pour communiquer l'idée d'éliminer différentes sortes de micro-organismes (germe, bactérie, virus, parasite dans ces contextes) uniquement. En outre, on note que désinfection (hyponyme) est une sorte de décontamination (hyperonyme) : T1 et T2 ont une relation d'hyperonymie-hyponymie en plus d'un lien de quasi-synonymie. 


\section{Post-tâche}

L'enseignant incite les apprenants à interagir et à partager leurs solutions et leurs déductions avec les autres étudiants de la classe, pour ensuite leur donner une rétroaction.

\section{$>$ Activité 2}

\section{Pré-tâche}

L'enseignant présente l'objet d'étude et les objectifs de l'exercice et donne des précisions nécessaires.

- Objet d'étude : la (quasi-)synonymie.

- Objectifs pédagogiques : organisation des connaissances et utilisation du terme juste suivant la situation de communication.

- Précision : à effectuer en équipe (p. ex. de 3) dans la salle de classe. Matériels disponibles : dictionnaires spécialisés, bases de données terminologiques, contextes extraits d'un corpus.

- Problèmes à résoudre :

a) Identifier et organiser les IC dans les contextes suivants de façon à distinguer les traits sémantiques des termes épuration et purification

- Contextes principaux :

(1) On effectue l' $\{$ épuration $\}$ des eaux usées non seulement pour protéger la santé de la population et éviter les maladies contagieuses, mais aussi [...].

(2) Ces produits peuvent contenir des agents complexants, [...], qui sont susceptibles de traverser les systèmes d' épuration $\}$ des eaux résiduaires.

(3) Les techniques abordées ci-dessous concernent principalement l'\{épuration $\}$ des eaux usées (eaux noires et eaux grises).

(4) Il existe des traitements plus ou moins complexes permettant l'\{épuration $\}$ des eaux grises.

- Contextes complémentaires :

(8) Les eaux usées [...] regroupent les eaux noires ou eaux vannes (eaux issues des sanitaires) et les eaux grises (eaux issues des éviers, lavabos, douches, baignoires, machines à laver, lave-linges).
(5) Dans les communes affectées, des produits de clarification et de \{purification\} de l'eau de boisson, des intrants pour le traitement des puits, du savon [...].

(6) Plusieurs types de procédés d'appoint adaptés à une utilisation domestique sont disponibles sur le marché pour la \{purification\} de l'eau du robinet.

(7) Les lampes UV utilisées dans les systèmes de \{purification\} de l'eau de laboratoire sont des lampes à mercure à basse pression.

(9) Au niveau d'une habitation, il existe plusieurs types d'eaux usées :

- les eaux vannes ou eaux noires en provenance des $W C[\ldots]$.

- les eaux ménagères ou eaux grises en provenance de la cuisine, des douches... 


\section{b) Compléter les énoncés suivants}

Énoncé 1

eaux noires | eau de robinet $\mid$ eaux de douches

Lorsque le chlore sert à la purification de l'/les

les THM en constituent un sous-produit inévitable.

Énoncé 2

eaux noires | eaux ménagères

La ville de Labelle a, sur la grande partie de son territoire, des résidences où l'épuration des . (ou grises) se fait par l'entremise d'une installation septique.

\section{Énoncé 3}

épuration | purification

Avec des filtres plantés auto-construits pour la/l' des eaux de la cuisine et de la salle de bains, ces procédés permettent de concevoir des maisons limitant au maximum leur consommation d'eau potable.

À ce stade, avant l'exécution de la tâche, l'enseignant peut présenter des activités préparatoires. Des contextes peuvent être fournis et des exercices d'organisation de données et de distinction de sens peuvent être effectués. Les étudiants se mettent en équipe et s'attribuent un rôle dans l'exécution de la tâche (voir l'activité 2).

\section{Exécution de la tâche}

\section{a) Identification et organisation des IC}

Les indices contextuels aident les apprenants à connaître la situation de communication dans laquelle les termes épuration et purification sont utilisés, ce qui les aide à employer le terme adéquat en contexte.

Pour trouver les IC, deux questions se posent :

1. Quel est le sens de $\mathrm{T} 1$ et $\mathrm{T} 2$ ? « ́́limination des matières nuisibles d'un milieu aquatique ».

2. De quel genre de milieu aquatique les matières nuisibles doivent-elles être éliminées? Le type de milieu aquatique constitue un IC.

Les IC se traduisent comme suit :

- Pour épuration :

- contexte (1) : eaux usées

- contexte (2) : eaux résiduaires

- contexte (3) : eaux usées (eaux noires, eaux grises)

- contexte (4) : eaux grises

- Pour purification :

- contexte (4) : eau de boisson

- contexte (5) : eau de robinet

- contexte (6) : eau de laboratoire

Les contextes supplémentaires (8 et 9) permettent de recueillir les renseignements suivants sur les types d'eaux usées :

- sortes d'eaux usées : eaux grises, eaux noires

- sortes d'eaux grises ou eaux ménagères : eaux provenant de la cuisine, des éviers,

de lavabos, de douches, de baignoires, de machines à laver, de lave-linges

- sortes d'eaux noires : eaux provenant des sanitaires

À cette étape, les étudiants organisent les données, ce qui va les aider à distinguer les traits sémantiques de $\mathrm{T} 1$ et $\mathrm{T} 2$. 
Figure 4. - Grille comparative d'épuration et purification.

\begin{tabular}{l|l|c}
\multicolumn{1}{c|}{ Épuration } & \multicolumn{1}{c|}{ Purification } & Indices contextuels \\
\hline $\begin{array}{l}\text { eaux usées }=\text { eaux résiduaires } \\
\text { eaux grises = eaux ménagères } \\
\text { (eaux de cuisine, des éviers, de } \\
\text { lavabos, de douches, de }\end{array}$ & $\begin{array}{l}\text { eau de boisson } \\
\text { eau de robinet } \\
\text { eauignoires, de machines à laver, } \\
\text { de lave-linges) }\end{array}$ & milieu aquatique \\
eaux noires $=$ eaux vannes & &
\end{tabular}

\section{b) Compléter les énoncés}

À présent les étudiants ont les informations nécessaires pour compléter les phrases cidessous.

\section{Énoncé 1}

Le contexte (9) indique que :

- eaux noires et eaux de douche s'emploient avec épuration

- eau de robinet s'emploie avec purification

eaux noires $\mid$ eau de robinet $\mid$ eaux de douches

Lorsque le chlore sert à la purification de l'eau de robinet, les THM en constituent un sous-

produit inévitable.

Énoncé 2

Premièrement, le contexte (9) indique que :

- eaux noires et eaux vannes renvoient au même concept

- eaux ménagères et eaux grises renvoient au même concept

Deuxièmement, dans l'énoncé (2), l'IC < également appelé > indique la synonymie

(voir section 2.2).

eaux noires leau ménagères

La ville de Labelle a, sur la grande partie de son territoire, des résidences où l'épuration des eaux ménagères (ou grises) se fait par l'entremise d'une installation septique.

\section{Énoncé 3}

Premièrement, le contexte (8) indique que : les eaux de la cuisine et eaux de salle de bain sont des sortes d'eaux grises (types d'eaux usées). Deuxièmement, les contextes (1), (2), (3) et (4) indiquent que les eaux usées, et donc eaux de la cuisine et eaux de salle de bain s'emploient avec épuration.

épuration | purification

Avec des filtres plantés auto-construits pour l'épuration des eaux de la cuisine et de la salle de bains, ces procédés permettent de concevoir des maisons limitant au maximum leur consommation d'eau potable.

\section{Post-tâche}

L'enseignant invite les apprenants à remettre par écrit leur solution en justifiant leur réponse: décrire leur réflexion et leur démarche, par exemple. Puis il les amène à partager leurs solutions avec les autres étudiants de la classe, pour ensuite leur donner une rétroaction.

\section{Conclusion}

Cet article porte sur la création d'activités lexicales basées sur corpus destinées aux étudiants en traduction. Ayant constaté que, malgré son importance en langue de 
spécialité, l'enseignement du lexS n'occupe pas la place qu'il devrait dans la formation de traducteurs, nous avons pour objectif de développer des méthodes d'enseignementapprentissage du lexique selon l'APT.

Par ailleurs, les développements en matière de l'exploitation du corpus et de conception d'outils d'interrogation de corpus aux fins d'enseignement nous encouragent à effectuer cette étude sur l'utilisation du corpus pour créer des activités lexicales. Nous estimons que la recherche d'informations dans le corpus permet à l'apprenant de découvrir le sens et les spécifiés des termes, dont le comportement et l'usage de par ses recherches, ce qui favorise son autonomie.

Dans cet article, nous avons présenté quelques modèles d'activités pédagogiques portant sur la synonymie. Nous nous sommes appuyés sur l'approche par tâches pour concevoir ces exercices afin de permettre à l'apprenant de résoudre les problèmes de manière autonome par la réflexion et la recherche. Les exercices visent à aider les apprenants à appréhender/distinguer les sens des termes en contexte et à les utiliser correctement suivant la situation de communication. Nous avons montré que l'analyse des contextes obtenus par le corpus permet de recueillir des éléments contextuels qui peuvent servir d'indices contextuels sur le sens et l'usage des termes. À cet effet, nous avons montré comment l'apprenant peut parvenir à identifier ces IC afin de distinguer les similitudes ou les différences sémantiques entre des paires de termes sémantiquement proches et de les utiliser correctement suivant le contexte.

Pour vérifier les forces et les faiblesses de nos activités lexicales basées sur corpus et créées selon l'APT, nous avons mené une expérimentation dans un cours de terminologie auprès d'étudiants de premier cycle participant à un cours de Terminologie. À la suite de l'analyse des données, nous apporterons des changements et des ajustements à ces exercices de façon à ce qu'ils répondent aux besoins des apprenants.

\section{BIBLIOGRAPHIE}

AlCARAz Enrique \& Hughes Brian (2014), Legal Translation Explained, Manchester, UK : St Jerome Publishing.

ASSOCIATION QUÉBÉCOISE DES TECHNIQUES DE L'EAU, Dictionnaire de l'eau, Québec, Canada : L'éditeur officiel du Québec.

BAKER Mona (1999), « The Role of Corpora in Investigating the Linguistic Behaviour of Professional Translators », International journal of corpus linguistics, 4(2), 281-298.

BeEby Allison, Rodríguez Inés Patricia \& SÁnchez-GiJón Pilar (2009), Corpus Use and Translating. Corpus Use for Learning to Translate and Learning. Corpus Use to Translate, Amsterdam, Pays Bas : John Benjamins Publishing.

BÉDARD Claude (1986), La traduction technique : principes et pratique, Montréal, Canada : Linguatech. 
BINON Jean \& VERLINDE Serge (2004), « L'enseignement/apprentissage du vocabulaire et la lexicographie pédagogique du français sur objectifs spécifiques (FOS) : le domaine du français des affaires ", Études de linguistique appliquée, 3, 271-283.

Binon Jean, DANCETTE Jeanne \& VerLINDE Serge (1998), « Comment améliorer le traitement des synonymes dans un dictionnaire de langue », T. Fontenelle, P. Hiligsmann, A. Michiels, A. Moulin \& S. Theissen (dir.), Actes du $8^{e}$ Congrès international de lexicographie : Euralex'98, Liège, Belgique : Université de Liège, 77-86.

Boulton Alex (2008), « Esprit de corpus : promouvoir l'exploitation de corpus en apprentissage des langues », Texte et Corpus, 3, 37-46.

Boulton Alex \& TYNe Henri (2014), Des documents authentiques aux corpus: démarches pour l'apprentissage des langues, Paris, France : Didier.

BOWKeR Lynne (2002), Computer-Aided Translation Technology: A Practical Introduction, Ottawa, Canada : University of Ottawa Press.

BOWKER Lynne \& PEARSON Jennifer (2002), Working with Specialized Language : A Practical Guide to Using Corpora, Londres, UK : Routledge.

BUREAU DE LA TRADUCTION (2006), Termium Plus, en ligne sur <www.btb.termiumplus.gc.ca> (12 décembre 2017).

CaValla Cristelle \& Loiseau Mathieu (2013), « Scientext comme corpus pour l'enseignement », A. Tutin \& F. Grossmann (dir.), L'écrit scientifique : du lexique au discours. Autour de Scientext, Rennes, France : Presses universitaires de Rennes, 163-182.

CAZEVIEILLE Françoise (2007), « Introduire le lexique spécialisé dès l'initiation en français scientifique ", Didáctica: Lengua y Literatura, 19, 173-185.

CHAMBERS Angela (2010), «L'apprentissage de l'écriture en langue seconde à l'aide d'un corpus spécialisé ", Revue française de linguistique appliquée, 15(2), 9-20.

CHETOUANi Lamria (1997), Vocabulaire général d'enseignement scientifique (VGES), Paris, France : L'Harmattan.

Condamines Anne \& Dehaut Nathalie (2011), « Mise en œuvre des méthodes de la linguistique de corpus pour étudier les termes en situation d'innovation disciplinaire : le cas de l'exobiologie », Meta : Journal des traducteurs, 56(2), 266-283.

DictionNAIRE ENVIRONNEMENT, en ligne sur <www.dictionnaire-environnement.com/>

(12 décembre 2017).

DuBuc Robert (1985), Manuel pratique de terminologie, Brossard, Canada : Linguatech.

ECHEVERRI Álavaro (2008), Métacognition, apprentissage actif et traduction : l'apprenant de traduction, agent de sa propre formation, Berlin, Allemagne : Éditions universitaires européennes.

GARCia-Debanc Claudine, MASSERon Caroline \& Ronveaux Christophe (2013), Enseigner le lexique, Namur, Belgique : Presses universitaires de Namur.

GUÉVEL Zélie (2007), « Caractéristiques linguistiques et culturelles de la traduction spécialisée », É. Lavault (dir.), Traduction spécialisée : pratiques, théories, formations, Bern, Allemagne : P. Lang, 73-91.

GonZÁlez Davies Maria (2004), Multiple Voices in the Translation Classroom: Activities, Tasks and Projects, Amsterdam, Pays-Bas : J. Benjamins. 
GrossmanN Francis (2011), « Didactique du lexique : état des lieux et nouvelles orientations », Pratiques. Linguistique, littérature, didactique, 149-150, 163-183.

HURTADO ALBIR Amparo (2008), « Compétence en traduction et formation par compétences », TTR : traduction, terminologie, rédaction, 21(1), 17-64.

JoHnS Tim (1986), « Micro-Concord: A Language Learner's Research Tool », System, 14(2), 151-162. Johns Tim (1991), « Should You Be Persuaded: Two Samples of Data-Driven Learning Materials », T. Johns \& P. King (dir.), Classroom Concordancing, English Language Research Journal, 4, 1-16.

KIRALY Donald (2000), A Social Constructivist Approach to Translator Education, Manchester, UK : St Jerome Publishing.

KÜBLER Natalie (2003), « Corpora and LSP Translation », F. Zanettin, S. Bernardini \& D. Stewart (dir.), Corpora in Translator Education, 25-42.

KÜBLER Natalie (2014), « Mettre en œuvre la linguistique de corpus à l'université. Vers une compétence utile pour l'enseignement/apprentissage des langues? », Recherches en didactique des langues et des cultures : Les Cahiers de l'Acedle, 11(1), 37-77.

L'Hoмme Marie-Claude (2004), La terminologie : principes et techniques, Montréal, Canada : Presses de l'Université de Montréal.

LANDURE Corinne \& BOULTON Alex (2010), « Corpus et autocorrection pour l'apprentissage des langues ", Asp, 57, 11-30.

Li Defeng (2013), « Teaching Business Translation: A Task-Based Approach », The Interpreter and Translator Trainer, 7(1), 1-26.

MANGIANTE Jean-Marc (2002), « Place et rôle du lexique spécialisé dans les discours de français commercial et économique ", Recherche et pratiques pédagogiques en langues de spécialité : Cahiers de l'Apliut, 21(4), 27-39.

MANIEZ François \& THoIRon Philipe (2007), « La formation du traducteur/rédacteur médical au $21^{\mathrm{e}}$ siècle : outils et enjeux », É. Lavault (dir), Traduction spécialisée : pratiques, théories, formations, Bern, Allemagne : P. Lang, 189-207.

MAILlot Jean-René (1981), La traduction scientifique et technique, Paris, France : Eyrolles.

MEYER Ingrid (2001), « Extracting Knowledge-Rich Contexts for Terminography », Recent Advances in Computational Terminology, 2, 279.

NoNNON Élisabeth (2012), « La didactique du français et l'enseignement du vocabulaire, dans vingt ans de revues de didactique du français langue première », Repères : Recherches en didactique du français langue maternelle, 46, 33-72.

NunAN David (2004), Task-Based Language Teaching, Cambridge : Cambridge University Press.

OFFICE DE LA LANGUE FRANÇAISE (2006), Grand dictionnaire terminologique, en ligne sur < www.granddictionnaire.com> (2 décembre 2012).

PeARson Jennifer (1998), Terms in Context, Amsterdam : John Benjamins Publishing.

PHAL André \& BEIS Lucette (1971), Vocabulaire général d'orientation scientifique : part du lexique commun dans l'expression scientifique, Paris : Centre de recherche et d'études pour la diffusion du français (CREDIF).

PHAL André, BeIs Lucette \& GougENHeIM Georges (1976), Vocabulaire général d'orientation scientifique (VGOS) : part du lexique commun dans l'expression scientifique, Paris, France : Didier. 
Ploux Sabine \& VICTORRI Bernard (1998), « Construction d'espaces sémantiques à l'aide de dictionnaires de synonyme », Traitement automatique des langues, 39(1), 161-182.

POLGUÈRE Alain (2003), Lexicologie et sémantique lexicale : notions fondamentales, Montréal, Canada : Presses de l'Université de Montréal.

ROULEAU Maurice (2011), La traduction médicale : une approche méthodique, Montréal, Canada : Linguatech.

SCARPA Federica (2010), La traduction spécialisée : une approche professionnelle à l'enseignement de la traduction, Ottawa, Canada : University of Ottawa Press.

SKEHAN Peter (2011), A Cognitive Approach to Language Learning, Oxford, UK : Oxford University Press.

TRÉVILLE Marie-Claude (2000), Vocabulaire et apprentissage d'une langue seconde : recherches et théories, Québec, Canada : Éditions Logiques.

TYNE Henry (2012), « Corpus Work with Ordinary Teachers: Data-Driven Learning Activities », J. Thomas \& A. Boulton (dir.), Input, Process and Product: Developments in Teaching and Language Corpora, Brno, République tchèque : Masaryk University Press, 114-129.

WILLIS Jane (1996), A Framework for Task-Based Learning, Londres, UK : Longman.

ZANETTIN Federico (2002), « Corpora for Translation Practice », E. Yuste-Rodrigo (dir.), Language Resources for Translation Work and Research, LREC 2002 Workshop Proceedings, Las Palmas de Gran Canaria : Espagne, 10-14.

ZANetTin Federico, Bernardini Silvia \& STEWARt Dominic (dir.) (2003), Corpora in Translator Education, Manchester, UK : St Jerome Publishing.

\section{NOTES}

1. Dans cette approche, l'enseignant remet des textes aux étudiants qui doivent le traduire pour le cours suivant; puis, dans une séance postérieure, il leur demande de faire une traduction collective et de partager leurs propositions de traduction.

2. Notre étude est circonscrite principalement au contexte canadien.

3. Par exemple, les cours de Langue et notions scientifiques et techniques, Langues et notions juridiques, Langue et notions économiques, qui figurent parmi les cours obligatoires en premier cycle de traduction.

4. Notre corpus contient des textes renfermant le lexique de la pollution de l'eau qui comprend la terminologie, mais aussi le vocabulaire général d'orientation scientifique (Phal, 1971) et le vocabulaire général d'enseignement scientifique (Chetouani, 1989).

5. GDT, en ligne sur <www.granddictionnaire.com/ficheOqlf.aspx?Id_Fiche=2071468> (12 décembre 2017); Termium, en ligne sur <www.btb.termiumplus.gc.ca/tpv2alpha/alphafra.html?lang=fra\& $\mathbf{i}=1 \&$ srchtxt=purification\&index=alt\&codom2nd_wet=1 - $\quad$ resultrecs $>$ (12 décembre 2017).

6. Dans les exemples et les exercices de ce texte, le terme clé est indiqué entre les signes \{\} et les IC, entre les signes <>. 


\section{RÉSUMÉS}

Cet article présente des activités lexicales créées à partir d'un corpus spécialisé pour les étudiants en traduction. Le lexique spécialisé est un élément clé qu'un traducteur doit maîtriser pour exprimer des connaissances liées à un domaine spécialisé. Pourtant, celui-ci n'occupe pas la place qu'il devrait dans la formation des traducteurs spécialisés. Afin de combler cette lacune, dans cet article, nous nous intéressons à la création d'activités basées sur un corpus. Nous nous reposons sur l'apprentissage par tâches pour concevoir des activités permettant aux apprenants de résoudre les problèmes lexicaux rencontrés au cours d'une traduction de façon autonome. Notre objectif est d'aider les apprenants à appréhender et à distinguer les sens des termes et à les utiliser correctement selon le contexte. Dans le présent article, nous nous concentrons sur les relations lexicales, particulièrement sur la synonymie.

This articles suggest corpus-based activities for translation students. The specialized lexicon is a key issue that a translator should master in order to express knowledge related to a specific field. However, the lexicon does not occupy the place that it should in translator training. In order to bridge the gap between translator training and professional translation requirements, we suggest corpus-based activities. We rely on a task-based learning approach in order to create activities that enable learners to solve problems related to the lexicon they might encounter when translating in an autonomous way. We aim to help learners to understand and distinguish the meaning of terms and to use them correctly in context. In this paper, we focus on lexical relations problems, more specifically (near-)synonyms.

\section{INDEX}

Keywords : translator training, specialized language, specialized lexicon, task-based approach, corpus, corpus analysis, lexical relations

Mots-clés : formation des traducteurs, langue de spécialité, lexique spécialisé, approche par tâches, analyse de corpus, relations lexicales

\section{AUTEUR}

\section{MARJAN ALIPOUR}

Université de Montréal, Département de linguistique et de traduction, Observatoire de linguistique Sens-Texte (OLST)

marjan.alipour@umontreal.ca 